Heavy ion reactions provide the first example for the application of quantum statistics to finite non-equilibrium systems. The study of "cold" (non-thermalized) fusion and fission processes, interesting in itself, is also relevant to the production of new transuranium elements and possibly even superheavynuclei. And in supercritical nuclear systems $\left(Z_{1}+Z_{2}>137\right)$, position creation has been observed which is a signal for new phenomena such as the formation of superheavy nuclear molecules.

Eventually our colleague on his round trip would get involved in the discussion of the consequences of the discoveries in particle physics. How do quark degrees of freedom manifest themselves in a bound system of nucleons? How can the mesonic description of nuclear forces be embedded in the evolving theory of strong interaction? Are there many body forces? How can the shellmodel description be reconciled with a picture of quark-bags in the nucleus? Clearly, the perspectives are changing considerably. In the past, the nucleus could be considered as a system of bound nucleons in their ground-state in a similar way that molecules and crys- tals are systems of atoms in their ground state. Through higher energies and probes it now becomes possible, however, to excite quark structures inside a bound nucleon. Thus the nucleus has to be described in more general terms as a multibaryon system. It is like going from the phonon spectrum of solids to the study of electronic excitations, which, of course, are different from those of isolated atoms. These effects may be studied by electromagnetic probes like electrons and muons or by hadronic probes like $\mathrm{K}$ mesons which allow a single nucleon inside a nucleus to be marked with a strange quark in a way quite similar to atoms being marked in a molecule by a radioactive tracer-atom.

In the last analysis, a nucleus would be described as a system, not of nucleons, but of quarks and gluons, although this makes little sense except for very high energy densities. The road to study such extreme conditions of matter is opened up by the developments in heavy ion physics. Heavy ions are not only a tool to excite spectroscopically interesting states and to produce new nuclides far off stability but their collisions allow us also to study the global properties of nuclear matter. First steps in this direction have been taken, but much higher heavy ion energies than presently available will be needed to force the quark-bags in a nucleus to overlap and to form a quarkgluon-plasma. Such states of matter may have existed in the first micro seconds after the beginning of the Universe.

Unavoidably our visitor would be confronted with many ideas and proposals for new facilities which are needed to take the next steps into the strange world of nuclei and their substructure. The talk would probably be about a multi$\mathrm{GeV}$ high intensity electron accelerator, high precision beams for intermediate energies, relativistic heavy ion beams, the secondary beams of high-intensity proton-accelerators and $\mathrm{K}$-meson factories. He realizes that bringing to life such projects in Europe would certainly depend on a substantial degree of international cooperation. For the moment, however, our returning colleague decides to read in more detail this present issue of Europhysics News to get more insight into some of the problems he has just heard about.

\title{
Spin Vibrations in Nuclei
}

\author{
Carl Gaarde, Roskilde \\ (The Nields Bohr Institute, University of Copenhagen)
}

Experiments over the last few years have led to a major breakthrough in our understanding of spin excitations in nuclei. The experiments have shown that spin-spin interactions between nucleons inside the nucleus build up correlations and give rise to collective motion of many nucleons.

These findings are another demonstration of the richness of structure in the nuclear spectrum. Collective effects are observed in a variety of ways. The socalled giant resonances are examples of collective motion and have been observed in many channels: surface- and pairing vibrations, compression modes, to mention a few.

One of the first resonances to be studied in detail was the giant dipole resonance, a collective vibration of protons against neutrons. A very selective probe for the study of this mode has been the absorption of light of the appropriate wavelength.
A probe with an unexpected specificity for spin excitations has been the $(p, n)$ reaction at intermediate energies. The incoming proton exchanges charge with the target and continues as a neutron, or, if we talk of protons and neutrons as nucleons with different isospin projection, we may also say, that isospin is transferred in the reaction. Recent experiments also show that spin is preferentially transferred; most of the outgoing neutrons have a spin direction different from that of the incoming proton.

An interesting aspect of the study of spin modes is the possible role of the internal structure of the nucleons. The simplest excitation of the nucleon is the $\triangle$-resonance with a mass of $1232 \mathrm{MeV}$, spin $S=3 / 2$ and isospin $T=3 / 2$. In a quark model, the $\Delta$ is an isospin-spin excitation of the nucleon wherein a dquark is transformed into a u-quark. We could therefore expect that in a process involving isospin-spin excitations the $\triangle$ resonance would also be excited.

\section{The $(p, n)$ Experiments}

The $(p, n)$ experiments have been performed at the Indiana University Cyclotron Facility using proton beams of energies between 100 and $200 \mathrm{MeV}$ bombarding targets of thickness typically $50 \mathrm{mg} / \mathrm{cm}^{2}$. The outgoing neutrons are detected, after a flight in air of around $100 \mathrm{~m}$, in large plastic detectors $15 \times 15 \times 100 \mathrm{~cm}^{3}$. A measurement of the time of flight, derived as the time difference between a beam burst and arrival in the detector, determines the energy of the neutron. Sub-nanosecond timing is achieved and corresponds to an energy resolution of $500 \mathrm{keV}-1 \mathrm{MeV}$ depending on energy and flight path. The scattering angle is changed in a beam swinger system by changing the direction of the beam impinging on the target. Despite the very small solid angle, the cross-section for the states we study is so large, it takes only about $15 \mathrm{~min}$ to get a spectrum. It has therefore been possible in rather short time to 


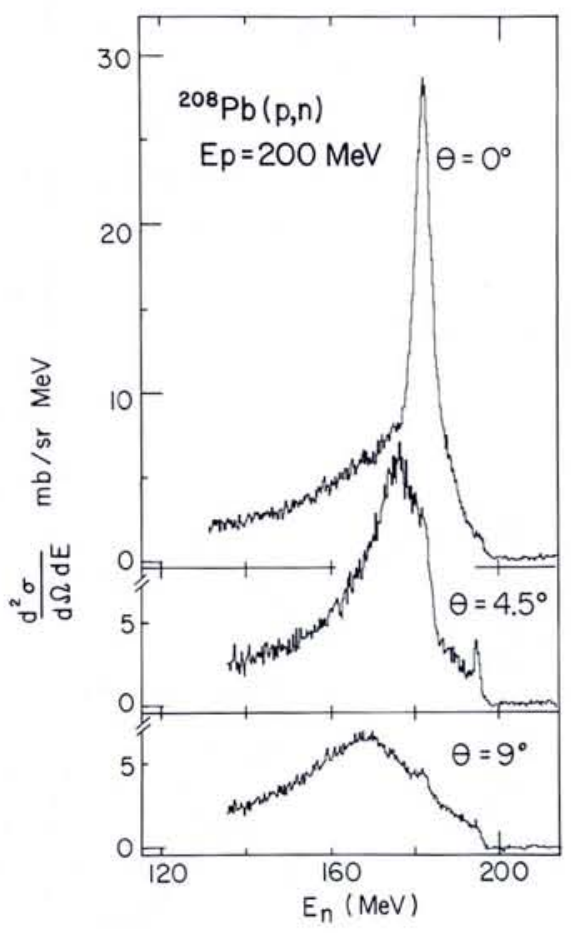

Fig. 1 - Neutron spectra at different angles from the proton bombardment of ${ }^{208} \mathrm{~Pb}$ at $200 \mathrm{MeV}$.

obtain systematic data for many nuclei at several energies.

In Fig. 1 are shown spectra at different angles from the $(p, n)$ reaction on ${ }^{208} \mathrm{~Pb}$. The zero degree spectrum is dominated by a single peak that has already become small in the $\theta=4.5^{\circ}$ spectrum. This spectrum is now dominated by another somewhat broader peak at a lower neutron energy (i.e. higher excitation energy) whereas in the $\theta=9^{\circ} \mathrm{spec}$ trum, this latter peak is small, and an even broader structure at still lower energy dominates.

The peak in the zero degree spectrum is interpreted as a collective excitation, involving transitions of neutrons into protons with simultaneous change of direction of their spin. Since the transition operator for $\beta$-decay with spin flip, $\sigma_{\mu} t_{-}$, transforming a neutron into a proton with change of spin direction, is referred to as the Gamow-Teller operator, these collective states are called Giant Gamow-Teller resonances. For the nucleus ${ }^{208} \mathrm{~Pb}$ with 82 protons and 126 neutrons, the 44 excess neutrons can through G-T transitions be transformed into protons and flip their spin. When we therefore use the words Giant GamowTeller state, we are implying that the peak we see in our zero degree spectrum corresponds to a transition where most of the 44 excess neutrons coherently change their direction in isospin-space, i.e. transform into protons and change their spin direction.
The $(p, n)$ spectra show very small cross-sections for transitions to states where spin transfer is not allowed, e.g. $\mathrm{O}^{+} \rightarrow \mathrm{O}^{+}$transitions. The spectra given in Fig. 1 are therefore totally dominated by spin transfer processes.

We implied above that the $(p, n)$ reaction measures Gamow-Teller strength. This has been demonstrated simply by measuring the $(p, n)$ cross-sections for a number of transitions for which the $\beta$ decay has been studied. From the lifetime and energy of the $\beta$-decay the strength can be deduced, and a comparison with the $(p, n)$ zero degree crosssections shows a strict proportionality when these are corrected for dependency on $A$ and excitation energy. We can therefore, from a $(p, n)$ cross-section deduce the Gamow-Teller strength for a given transition.

Another interesting aspect of the $(p, n)$ reaction is that we can calculate the cross-section using the interaction between free nucleons as the interaction between the incoming proton and a nucleon in the target. In doing this, we are assuming that the reaction is a one step process. This is a good approximation around $200 \mathrm{MeV}$ where the mean free path for nucleons in nuclei is maxi$\mathrm{mal}$, and the effect of other nucleons on the incoming proton and outgoing neutron is minimal.

\section{Spin Densities}

The peaks in the spectra in Fig. 1 can also be described as corresponding to vibrations in isospin-spin space. The peak in the zero degree spectrum is the simplest mode, the 44 excess neutrons flip their spin and become protons, but with no change in orbital motion. On the microscopic level, an example could be a neutron moving in an $i_{13 / 2}(\ell=6 ; j=\ell+$ $1 / 2$ ) orbit being transformed into a proton in an $i_{11 / 2}(\ell=6 ; j=\ell-1 / 2)$ orbit. The proton particle and the neutron hole couple to $J^{\pi}=1$ and positive parity.

The spin density for this $\Delta \ell=0$ mode is shown in Fig. 2. The figure can be thought of as a snapshot of the nucleus, with the spin density almost homogeneous over its volume, as in a ferromagnet. This isospin-spin field is a result of correlations built up by particle-hole interactions which line up the spins of many nucleons.

The peak in the $4.5^{\circ}$ spectrum in Fig. 1 is interpreted as an envelope of three giant resonances with spins 0,1 and 2 and negative parity. The angular distribution of the peak determines the transferred angular momentum to be $\Delta \ell=1$. In a microscopic description, the excitation could, for example, be a transition from a $h_{11 / 2}(\ell=5 ; j=\ell+1 / 2)$ neutron orbit to an $i_{11 / 2}(\ell=6 ; j=\ell-1 / 2)$ proton orbit. This is a transition from one shell into the next. In ${ }^{208} \mathrm{~Pb}$, the energy difference between shells is $6 \mathrm{MeV}$, consistent with the resonance energy being 6 $\mathrm{MeV}$ larger than for the $\ell=0$ resonance. The spin density for the three collective states is shown in Fig. 2, where the pattern is seen to be more complex. These modes involve even more nucleons not only the 44 excess neutrons but also the neutrons in the next lower shell are correlated. For example, in ${ }^{208} \mathrm{~Pb}$, the spin densities for the $\Delta \ell=1$ vibrations as given in Fig. 2 are built from correlations among more than 80 neutrons a truly collective effect!

\section{Sumrule}

A very general sumrule applies for Gamow-Teller transitions: the difference between the sum of $\beta$ strengths for $\beta$. and $\beta_{+}$transitions from some initial state

$$
S_{\beta-}-S_{\beta+}=3(N-Z) .
$$

The sum is made over all final states in both the $\beta_{-}$and $\beta_{+}$channels.

Fig. 2 - Spin densities for the collective states indicated in Fig. 1 are shown as cuts through the nucleus containing the $z$-axis (except for the $1^{-}$mode where the cut is in the $x$-y plane). All are axially symmetric about the $z$-axis. The $1^{+}$density corresponds to the $\theta=0^{\circ}$ peak whereas the peak at $\theta=4.5^{\circ}$ is an envelope of peaks with densities of $\mathrm{O}^{-}, 1^{-}, 2^{-}$.

$1^{+}$

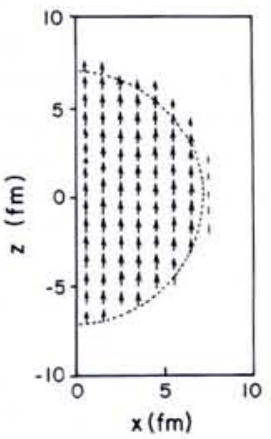

$0^{-}$

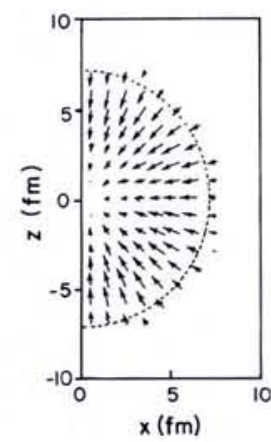

$1^{-}$

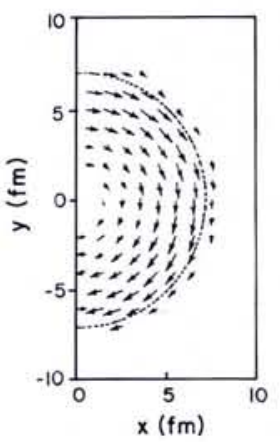

$2^{-}$

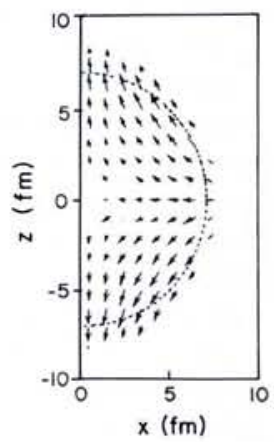


The only assumption in deriving this expression is that the $\mathrm{G}-\mathrm{T}$ operator only changes the direction of nucleons in isospin- and spin-space, and the nucleus behaves as if it were composed of nucleons in free space.

Sumrules have been useful tools in the study of giant resonances as they provide a natural unit for coupling strength. A state is only a giant resonance if a significant part of the sumrule is concentrated on the state. Sumrules are also in general less model-dependent than other quantities. Thus the sumrule given above is independent of the interaction responsible for the correlated motion.

We mentioned that the $(p, n)$ reaction measures $\beta$ _ G-T strength and therefore also the total $\beta$ _ strength. To use the sumrule we need to know $S_{\beta+}$. First we note that $S_{\beta+}$ is a positive number, and the sumrule therefore gives a minimal value of $3(N-Z)$ for the $\beta$ _ strength. For nuclei with a large neutron excess, $S_{\beta+}$ is small because the final neutron states into which the $\beta$-decaying protons could decay are already occupied (Pauli blocked). The $S_{\beta+}$ can in specific cases be estimated from models, as even large errors in a small number give small total uncertainties in $S_{\beta-}$.

The analysis of the $(p, n)$ spectra shows that only between 50 and $65 \%$ of the $\beta_{\text {_ }}$ strength deduced from the sumrule is found in the expected energy regions of the spectra. In the ${ }^{208} \mathrm{~Pb}$ spectrum that means the region lower than and including the collective state.

Two explanations for the missing strength have been discussed:

i) Coupling to a more complicated structure removes strength from the low lying region and spreads it thinly over a large energy region. In the ${ }^{208} \mathrm{~Pb}$ spectrum the missing strength would then be in the long tail towards higher excitation energy, i.e., lower outgoing neutron energy. We have no indication of G-T strength in this tail region, so we cannot rule out the possibility.

ii) Coupling to the $\triangle$ resonance removes strength from the low energy region. The inclusion of the internal degrees of freedom for the nucleon, specifically the $\triangle$, changes the sumrule. Knowing the $\triangle$-nucleon interaction inside the nucleus, we can calculate how much strength is removed from the nuclear sector. Our sumrule is no longer modelindependent. In the constituent quark model for the nucleon, the quarks are fermions with isospin and spin in relative $s$-motion, and the $\Delta$ is an isospin-spin excitation of the nucleon. There is a definite relation between the nucleon-nu- cleon and the $\triangle$-nucleon interaction in the isospin-spin channel. In this model, $35 \%$ of the strength in the nuclear sector is removed. The reason for this large effect from an excitation $300 \mathrm{MeV}$ away is that all nucleons are involved whereas for the G-T collective state it is only $(N-Z)$ neutrons. The coupling to the $\triangle$ also gives rise to an admixture of $\triangle^{\prime} \mathrm{s}$ in nuclear ground states - $5 \%$ for the model above. So when we observe less than the $3(N-Z)$ sumrule strength, it is because we have to carry some $\triangle^{\prime}$ s along in the vibration.

\section{Direct $\triangle$-excitation}

In Fig. 3 triton spectra from $\left({ }^{3} \mathrm{He}, \mathrm{t}\right)$ reactions on carbon and hydrogen at a bombarding energy of $2 \mathrm{GeV}$ are presented. The $\left({ }^{3} \mathrm{He}, \mathrm{t}\right)$ reaction is a chargeexchange reaction like $(p, n)$ and shows the same selectivity for spin-transfer transitions as does the $(p, n)$ reaction at corresponding energies.

The experiments were performed at the Laboratoire National Saturne, Saclay, using a magnetic spectrometer to analyze the outgoing tritons. The spectrometer can bend up to $2 \mathrm{GeV}$ tritons, but such particles are rather stiff, and the spectrometer is by nuclear physics standards very large. It consists of 5 dipoles and 6 quadrupoles with a distance of $35 \mathrm{~m}$ between target and focal plane.

The spectrum from the proton target shows that the $\left({ }^{3} \mathrm{He}, \mathrm{t}\right)$ reaction very selectively excites the $\Delta^{++}$-state. The natural width of the $\triangle$ is $120 \mathrm{MeV}$, and the figure shows that there is very little yield outside the resonance. The $\left({ }^{3} \mathrm{He}, \mathrm{t}\right)$ reaction on the proton is therefore a good way to make a beam of $\Delta \mathrm{s}$. The only problem is that $\Delta s$ travel less than $2 \mathrm{fm}$ before they decay. The spectrum also strongly suggests that the $\Delta$ indeed is an isospin-spin excitation of the nucleon. The observed cross-section is well described as the result of exchanging a $\pi$ - and a $\rho$-meson, bosons that carry isospin and spin.

The triton spectrum from the carbon target shows a strong excitation of the $1^{+}$ground state in ${ }^{12} \mathrm{~N}$, (a Gamow-Teller transition) and a broad peak of width around $270 \mathrm{MeV}$. The nature of this broad structure is not well understood, but it could be quasifree $\triangle$ production, i.e. a nucleon in ${ }^{12} \mathrm{C}$ is transformed into a $\triangle$ in a plane wave final state. It could equally be an envelope of coherent $\triangle$ nucleon hole states with different spin parities. We have seen such an example in the $4.5^{\circ}$ spectrum in Fig. 1.

We conclude from the $\left({ }^{3} \mathrm{He}, \mathrm{t}\right)$ spectra shown here, that the $\triangle$ is an isospin-spin

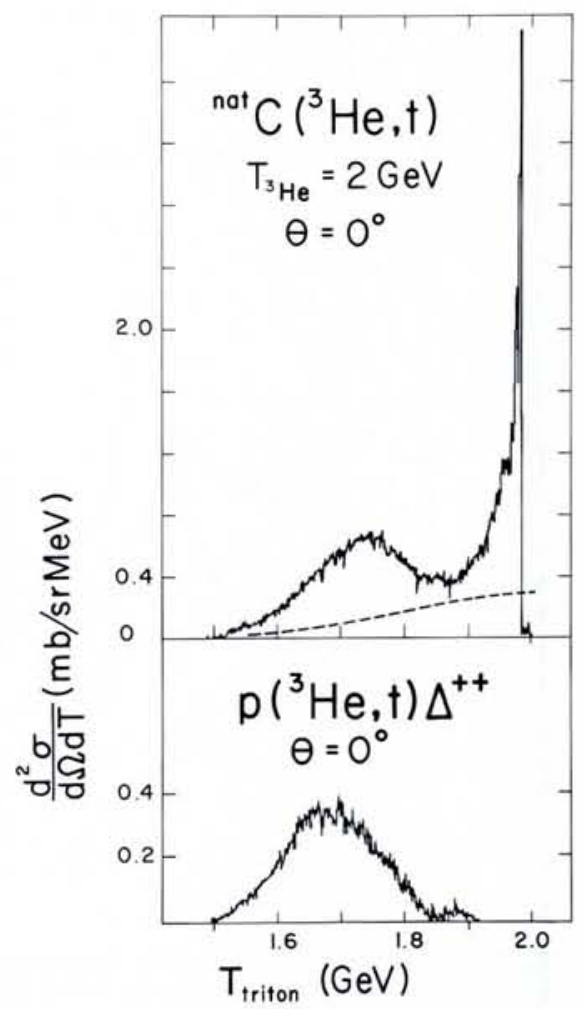

Fig. 3 - Triton spectra at $\mathrm{O}^{\circ}$ from the ${ }^{3} \mathrm{He}$ bombardment of natural carbon and hydrogen.

excitation of the nucleon, and that $\triangle \mathrm{ex}$ citations are observed in nuclei. We can however at present not relate the missing Gamow-Teller strength to the direct excitation of $\Delta s$ in nuclei. The $\left({ }^{3} \mathrm{He}, t\right)$ reaction seems to be a very good probe for the study of $\Delta s$ in nuclei.

\section{Summary}

$(p, n)$ experiments have shown that spin-spin interactions inside the nucleus give rise to correlated motion of many nucleons. Through normalization to $\beta$ decay it has been possible to deduce total $\beta$-decay strength from zero degree $(p, n)$ cross-sections, but comparison with the value derived from a very general sumrule shows missing Gamow-Teller strength. Inclusion of the internal degrees of freedom of the nucleon is an intriguing possibility to explain this.

The charge-exchange reactions at higher bombarding energies selectively excite the $\Delta$ resonance. The further study should hopefully help in our understanding of how $\Delta s$ behave in the nuclear medium, and the role in general of $\triangle \mathrm{s}$ in nuclear structure.

\section{REFERENCES}

Two review articles contain references to recent work in this field.

Goodman C., Gamow-Teller Resonances, Nucl. Phys. A374 (1982) 241c.

Gaarde C., Gamow-Teller and M1 Resonances, Nucl. Phys. A396 (1983) 127c. 